\section{Worse patient-reported outcomes and higher risk of reoperation and adverse events after total hip replacement in patients with opioid use in the year before surgery: a Swedish register-based study on 80,483 patients}

\author{
Johan SIMONSSON ${ }^{1,2}$, Erik BÜLOW ${ }^{1,3}$, Karin SVENSSON MALCHAU ${ }^{1}$, Fredrik NYBERG ${ }^{4}$, \\ Urban BERG ${ }^{1,2}$, and Ola ROLFSON ${ }^{1,3}$
}

1 Department of Orthopaedics, Institute of Clinical Sciences, Sahlgrenska Academy, University of Gothenburg, Gothenburg; ${ }^{2}$ Department of Surgery and Orthopaedics, Kungälvs Hospital, Kungälv; ${ }^{3}$ Swedish Arthroplasty Register, Gothenburg, Centre of Registers Västra Götaland, Gothenburg; ${ }^{4}$ School of Public Health and Community Medicine, Institute of Medicine, Sahlgrenska Academy, University of Gothenburg, Gothenburg, Sweden

Correspondence: Johan.s.simonsson@vgregion.se

Submitted 2021-07-15. Accepted 2021-11-19.

Background and purpose - Recent studies indicate that preoperative use of opioids could be associated with higher rates of complications and worse patient-reported outcomes (PROs) after orthopedic surgery. We investigated the prevalence of preoperative opioid use and analyzed its influence on risk of revision, adverse events (AE), and PROs in patients with total hip replacement (THR).

Patients and methods - This observational study included 80,483 patients operated on in 2008-2016 with THRs due to osteoarthritis. Data was obtained from the Swedish Hip Arthroplasty Register, Statistics Sweden's sociodemographic registers, the Swedish National Patient Register, and the Prescribed Drug Register. We focused on patients with $\geq 4$ opioid prescriptions filled 1 year prior to THR. To control for confounding, we used propensity scores to weight subjects in our analyses. Logistic and linear regression was used for outcome variables with adjustments for sociodemographic variables and comorbidities.

Results - Patients with $\geq 4$ opioid prescriptions in the year before THR $(\mathrm{n}=14,720[18 \%])$ had a higher risk of revision within 2 years (1.8\% vs. $1.1 \%$ OR $1.4,95 \%$ CI $1.3-$ 1.6) and $\mathrm{AE}$ within 90 days (9.4\% vs. $6.4 \%$ OR $1.2,95 \% \mathrm{CI}$ 1.2-1.3) compared with patients without opioid treatment in the preoperative period. Patients with $\geq 4$ opioid prescriptions rated 5 points worse on a $0-100$ scale of Pain Visual Analogue Scale (VAS) and 9 points worse on a general health (EQ) VAS 1 year postoperatively.

Interpretation - Having $\geq 4$ opioid prescriptions filled in the year before surgery is associated with a higher risk of revision, adverse events, and worse PROs after THR. Consequently, preoperative opioid treatment should be addressed in the clinical assessment of patients eligible for THR.
A large proportion of patients eligible for THR use analgesic medication in the period before proceeding with surgery (1). Opioids have been shown to have limited effect on chronic pain and are not recommended for OA patients according to international guidelines. Nevertheless, a recent study indicated that $20-25 \%$ of hip or knee OA patients in Sweden have been treated with 1 or more opioid prescriptions during a 12 -month period $(2,3)$. The general side effects of opioids are well documented and several studies have focused on preoperative opioid use as a possible risk factor for worse outcomes after orthopedic surgery, especially after joint replacement $(4,5)$. It has been reported that patients with higher use of opioids in the preoperative period are at greater risk of complications and early reoperation. They also have a higher rate of residual pain after surgery (6). The rate of preoperative opioid use in Swedish patients undergoing THR due to OA, and its influence on outcomes, has not been studied.

We explored the preoperative opioid use in patients undergoing THR and analyzed its effects on postoperative outcomes, to answer our research questions:

- What is the rate and pattern of preoperative opioid prescribing in patients undergoing THR due to OA in Sweden?

- What is the influence of opioid treatment in the year before THR on outcomes after THR?

\section{Patients and methods}

\section{Study population}

We conducted an observational register-based study. The studied cohort consisted of all patients 18 years or older from the Swedish Hip Arthroplasty Register (SHAR) operated on with 


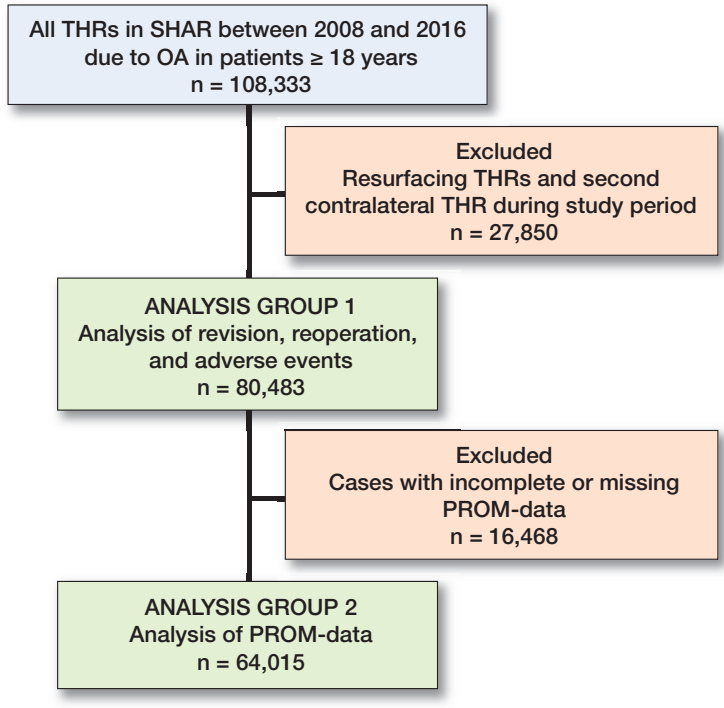

Included patients. $\mathrm{THR}=$ total hip replacement. $\mathrm{OA}=$ osteoarthritis. $\mathrm{PROM}=$ patient-reported outcome measure

THR from 2008 to 2016 due to OA (all M16.X according to ICD-10 codes). In patients receiving bilateral THRs during the study period, the first THR was the one analyzed in order to have only independent observations. Resurfacing hip replacements were excluded, and in the analyses of patient-reported outcomes (PROs), patients with incomplete PRO registrations at baseline or 1 year postoperatively were excluded (Figure).

\section{Data sources}

Patient-specific variables such as age, sex, ASA score, BMI, and Charnley class in addition to surgical variables such as fixation method (cemented, uncemented, hybrid, reverse hybrid) and surgical approach (posterior, direct lateral) were collected from the SHAR. Further patient information was obtained from registers held by Statistics Sweden and the National Board of Health and Welfare. Data from the different registers was linked via personal identity number (7). Information on filled opioid prescriptions came from the Prescribed Drug Register. ICD-10 diagnoses and Nordic MedicoStatistical Committee (NOMESCO) Classification of Surgical Procedures (NCSP) codes registered in specialized inpatient and outpatient healthcare were collected from the Swedish National Patient Register (NPR) and used to calculate the Elixhauser comorbidity index and to define postoperative adverse events (8-10). Socioeconomic variables were gathered from the Longitudinal Integrated Database for Health Insurance and Labour Market Studies (LISA) (11). For descriptive tables, BMI was categorized using WHO cutoffs but combining the lowest 2 and highest 2 categories due to very few patients with underweight and class 3 obesity ( -24.9 as underweight and normal weight, 25-29.9 as overweight, 30-34.9 as class 1 obesity, $\geq 35$ as class 2 and 3 obesity). Age was categorized in line with cutoffs used in the SHAR annual report (12).
Table 1. Filled opioid prescriptions in 1-year period before total hip replacement among adult osteoarthritis patients. Values are number of patients (\%)

\begin{tabular}{lcc}
\hline Prescriptions & Analysis group 1 & Analysis group 2 \\
\hline Any opioid & $35,272(44)$ & $27,374(43)$ \\
$\geq 4$ of any opioid & $14,720(18)$ & $11,076(17)$ \\
Any weak opioid & $27,652(34)$ & $21,649(34)$ \\
$\geq 4$ of weak opioid & $9,478(12)$ & $7,270(11)$ \\
Any strong opioid & $14,522(18)$ & $10,873(17)$ \\
$\geq 4$ of strong opioid & $5,354(7)$ & $3,819(6)$ \\
\hline
\end{tabular}

Patients can be in both weak and strong opioid groups. There is an overlap between the groups.

For socioeconomic data, education level was defined as low ( 9 years or less in school), middle (high school level), and high (tertiary education). Income levels were defined as low (below lowest quartile, Q1), middle (the interquartile range, between Q1 and Q3), and high (above highest quartile, Q3) from the disposable income per consumption unit of the whole cohort.

\section{Exposures}

The primary exposure of this study was filled prescriptions for opioid medications. We defined opioids by the Anatomical Therapeutic Chemical (ATC) code N02A with all available sub-categories. Weak opioids, mainly codeine and tramadol analgesics, were defined as ATC codes N02AJ06, N02AJ09, and N02AX02. All other N02A codes were classified as strong opioids (Table 1). For further analysis we combined weak and strong opioids and only considered total number of prescriptions, regardless of opioid strength. With great variations in adherence to prescription recommendations and many packages of weak opioids containing more oral morphine equivalents (OMEQs) than packages of strong opioids we cannot make assumptions on actual opioid exposure based on prescriptions being weak or strong opioids. We used the measure of $\geq 4$ filled opioid prescriptions in the 1-year period before THR as a proxy of repeated or long-term opioid use. We chose a cut-off at 4 prescription iterations as a likely level where ingestion has actually occurred, with the possibility of 1,2 , or 3 prescriptions being test prescriptions or prescriptions meant for postoperative use, filled preoperatively. We also included groups who had 1,2, or 3 opioid prescriptions filled in the year prior to THR in order to have a crude indication of a possible dose-response relationship.

\section{Outcome measures}

The outcome variables in analysis of group 1 (Figure) were revision within 2 years, reoperation within 2 years, and adverse events within 90 days. Revision was defined as a procedure where 1 or multiple prosthetic components were replaced, added, or removed and reoperation as any procedure relating to the operated joint without any exchange of prosthetic parts. Adverse events were defined according to the 
definition used in the SHAR's annual report as occurrence of specified ICD-10 or the NOMESCO Classification of Surgical Procedures (NCSP) codes identified as possible complications to THR in the NPR within 90 days of surgery. In analysis of group 2, PRO data collected from the SHAR via patient-filled questionnaires was analyzed. Variables were: Pain VAS, EQ VAS, and EQ-5D-SE (hip pain Visual Analogue Scale [VAS], EuroQoL 5-dimensional health status questionnaire, Swedish experience-based). Pain, satisfaction, and EQ (general health) scores are on a 0 to 100 -point visual scale. EQ-5D-SE is an index of 5 health domains with 3 possible levels each. SE signifies the use of the Swedish Experience-based value set to calculate the index values (13).

\section{Statistics}

Each patient could have been treated with $0,1,2,3$, or $\geq 4$ opioid prescriptions. We assumed that these treatment groups were non-random and related to severity of symptoms, comorbidities, and physician preferences. We also speculated that patients with less severe symptoms could end up with better outcomes. Hence, there was an obvious risk of confounding involving treatment and outcome. We therefore used propensity scores to re-weight each patient's impact on the estimates in the regression analyses. First, propensity scores were defined as the probability of each patient being assigned the treatment he or she was observed to receive. This probability was estimated from data which was available prior to the studied outcomes (diagnosis, age, sex, BMI, ASA score, education, civil status, disposable income per consumption unit, region of birth, Elixhauser comorbidity index, as well as earlier prescriptions of medications for depression, anxiety, and/or chronic pain). The relation between those covariates and the probability of received treatment was modeled by a generalized boosting method (GBM) with 2,000 trees using the R-package "WeightIt" (R Foundation for Statistical Computing, Vienna, Austria) (14). Missing data were handled by surrogate splitting and probability estimates outside an inner 95\% empirical interval were trimmed (ibid.). Reciprocals of the estimated treatment probabilities/propensity scores were then used for inverse probability weighting in the regression analyses of the desired outcomes. In this way, patients with a low propensity for their given treatment were up-weighted to address potential confounding of the estimated associations by a possibly strong correlation between treatment and the perceived need for such treatment.

All further analyses were performed in SPSS 25 (IBM Corp, Armonk, NY, USA). For the dichotomous variables of revision within 2 years, reoperation within 2 years, and adverse events within 90 days, we used simple and multiple logistic regression to calculate odds ratios (ORs) with their 95\% confidence intervals (CI). We considered odds ratios, in this context of low incidence and low odds ratios, a good approximation of relative risk. For the continuous variables of PRO data, linear regression analysis was used to calculate effect estimates of opioid treatment on each respective patient-reported outcome measure (PROM), with respective CI. The following variables were used for adjustment of regression analyses: age (continuous), sex, BMI (continuous), ASA score, Charnley class, fixation method (cemented, uncemented, hybrid, or reverse hybrid), Elixhauser comorbidity index, surgical approach (direct lateral or posterior), income level, and educational level (15-18). For analysis of PROM data, we also adjusted for preoperative values of the respective PROM variable as well as preoperative use of antidepressant medication (19).

\section{Ethics, data sharing, funding, and potential conflicts of interest}

This study was approved by the regional ethical review board in Gothenburg (271-14). This study involved data that exist in pseudoanonymized structured format with no way for researchers to connect personal patient information to specific research subjects. Data may be made available to other researchers upon request to registercentrum@vgregion.se, given that approval from the data owners, the National Board of Health and Welfare, Registercentrum Västra Götaland, and Statistics Sweden can be provided, as well as ethical approval from the Swedish ethical review authority. No intervention was made in this study and all study subjects were treated according to clinical practice at the time of surgery. Patients had the possibility, at any time, to opt out of being recorded in the SHAR. No financial support was received for this study. No competing interests were declared.

\section{Results}

In the study population $44 \%$ of patients undergoing THR had at least 1 opioid prescription filled in the year before surgery. $18 \%$ of patients had $\geq 4$ prescriptions filled during the same period. Female patients had $\geq 4$ filled opioid prescriptions to a higher degree than male patients (21\% of female vs. $15 \%$ of male). There was also a higher proportion of patients treated with $\geq 4$ prescriptions in patients with lower level of education, lower income, higher BMI, higher ASA score, and older age (Tables 2 and 3).

Compared with the opioid naive, patients with $\geq 4$ filled opioid prescriptions had a higher general rate of complications for all 3 outcome measures: for adverse events within 90 days $1,383(9.4 \%)$ vs. 2,899 (6.4\%) (adjusted OR 1.2, CI $1.2-1.3)$, for reoperations within 2 years $373(2.5 \%)$ vs. 702 (1.6\%) (adjusted OR 1.4, CI 1.3-1.5), and for revision within 2 years $261(1.8 \%)$ vs. $482(1.1 \%)$ (adjusted OR 1.4, CI 1.31.6) (Table 4).

Compared with the opioid naive, patients with $\geq 4$ opioid prescriptions rated higher on pain, lower on general health and EQ-5D-SE at baseline (69 vs. 59 Pain VAS, 48 vs. 58 EQ VAS, and 0.68 vs. 0.76 EQ-5D-SE; Table 5). They did not reach the absolute level of the opioid naive at 1 year post- 
Table 2. Analysis group 1: adverse events and reoperations. Patient characteristics at baseline grouped by number of filled opioid prescriptions among adult osteoarthritis patients with total hip replacement. Values are number of patients (\%)

\begin{tabular}{|c|c|c|c|c|c|c|}
\hline \multirow[b]{2}{*}{ Factor } & \multirow[b]{2}{*}{ Entire group } & \multicolumn{5}{|c|}{ Number of filled opioid prescriptions } \\
\hline & & 0 & 1 & 2 & 3 & $\geq 4$ \\
\hline Total observations & 80,483 & $45,211(56)$ & $10,785(14)$ & $5,885(7)$ & $3,882(5)$ & $14,720(18)$ \\
\hline \multicolumn{7}{|c|}{ (1) } \\
\hline Female & $45,442(57)$ & $23,754(53)$ & $6,428(60)$ & $3,562(61)$ & $2,369(61)$ & $9,329(63)$ \\
\hline Male & $35,041(43)$ & $21,457(47)$ & $4,357(40)$ & $2,323(39)$ & $1,513(39)$ & $5,391(37)$ \\
\hline \multicolumn{7}{|c|}{ (2) } \\
\hline$\leq 39$ & $679(1)$ & $378(1)$ & $87(1)$ & $39(61)$ & $34(1)$ & $141(1)$ \\
\hline $40-44$ & $1,021(1)$ & $594(1)$ & $140(1)$ & $73(1)$ & $48(1)$ & $166(1)$ \\
\hline $45-49$ & $2,323(3)$ & $1,256(3)$ & 332 (3) & $160(3)$ & $103(3)$ & 472 (3) \\
\hline $50-54$ & $4,294(5)$ & $2,420(5)$ & $601(6)$ & $282(5)$ & $196(5)$ & 795 (5) \\
\hline $55-59$ & $7,183(9)$ & $4,061(9)$ & $989(9)$ & $502(9)$ & $351(9)$ & $1,280(9)$ \\
\hline $60-64$ & $12,177(15)$ & $6,930(15)$ & $1,708(16)$ & $878(15)$ & $570(15)$ & $2,091(14)$ \\
\hline $65-69$ & 15,609 (19) & $9,216(20)$ & $1,960(18)$ & $1,061(18)$ & $714(18)$ & $2,658(18)$ \\
\hline $70-74$ & $14,826(18)$ & 8,529 (19) & $1,989(18)$ & $1,100(19)$ & 703 (18) & 2,505 (17) \\
\hline $75-79$ & $12,067(15)$ & $6,746(15)$ & $1,574(15)$ & $940(16)$ & $613(16)$ & $2,194(15)$ \\
\hline $80-84$ & $7,175(9)$ & 3,659 (8) & $997(9)$ & $564(10)$ & $381(10)$ & $1,574(1)$ \\
\hline$\geq 85$ & $3,129(4)$ & $1,422(3)$ & $408(4)$ & $286(5)$ & 169 (4) & $844(6)$ \\
\hline \multicolumn{7}{|l|}{ ASA score } \\
\hline 1 & $20,058(47)$ & $13,049(30)$ & 2,693 (35) & $1,319(23)$ & $753(20)$ & $2,244(16)$ \\
\hline 2 & $9,422(13)$ & 25,507 (58) & $6,252(50)$ & $3,388(60)$ & $2,274(60)$ & $8,487(60)$ \\
\hline$\geq 3$ & $27,965(40)$ & $5,186(12)$ & $1,464(14)$ & $982(17)$ & $736(20)$ & $3,491(24)$ \\
\hline \multicolumn{7}{|l|}{ BMI category } \\
\hline$\leq 24.9$ & $24,551(32)$ & $14,408(34)$ & 3,261 (32) & $1,778(32)$ & $1,073(29)$ & $4,031(29)$ \\
\hline $25-29.9$ & $33,259(43)$ & $19,268(45)$ & $4,522(44)$ & $2,319(41)$ & $1,596(43)$ & $5,554(40)$ \\
\hline $30-34.9$ & 14,286 (19) & 7,416 (17) & $1,879(18)$ & $1,147(20)$ & $774(21)$ & $3,070(22)$ \\
\hline$\geq 35$ & $4,426(6)$ & $1,898(4)$ & $593(6)$ & $374(7)$ & $257(7)$ & $1,304(9)$ \\
\hline \multicolumn{7}{|l|}{ Surgical approach } \\
\hline Lateral & $37,636(47)$ & $21,329(48)$ & $5,070(47)$ & $2,768(47)$ & $1,791(46)$ & $6,678(46)$ \\
\hline Posterior & $42,255(53)$ & $23,522(52)$ & 5,637 (53) & $3,083(53)$ & $2,073(54)$ & $7,940(54)$ \\
\hline \multicolumn{7}{|l|}{ Fixation method } \\
\hline Cemented & $52,434(65)$ & $28,814(64)$ & 7,007 (65) & $3,961(67)$ & $2,654(68)$ & $9,998(68)$ \\
\hline Uncemented & $15,330(19)$ & $9,188(20)$ & 2,035 (19) & 995 (17) & 665 (17) & 2,447 (17) \\
\hline Hybrid & $1,693(2)$ & $930(2)$ & $208(2)$ & $126(2)$ & $81(2)$ & $348(2)$ \\
\hline Reverse hybrid & $11,026(14)$ & 6,279 (14) & 1,535 (14) & $803(14)$ & 482 (12) & $1,927(13)$ \\
\hline \multicolumn{7}{|c|}{ Educational level } \\
\hline Low & $25,895(32)$ & $13,889(31)$ & $3,418(32)$ & $1,977(34)$ & $1,361(35)$ & $5,250(36)$ \\
\hline Middle & $33,201(42)$ & $18,430(41)$ & $4,476(42)$ & $2,456(42)$ & $1,638(43)$ & $6,201(42)$ \\
\hline High & $20,760(26)$ & $12,543(28)$ & $2,803(26)$ & $1,403(24)$ & $850(22)$ & $3,161(22)$ \\
\hline \multicolumn{7}{|l|}{ Income level } \\
\hline Low & $19,892(25)$ & $10,026(22)$ & $2,543(24)$ & $1,530(26)$ & $1,069(28)$ & $4,724(32)$ \\
\hline Middle & $40,095(50)$ & $22,509(50)$ & $5,374(50)$ & $2,885(49)$ & $1,943(50)$ & $7,384(50)$ \\
\hline High & $20,383(25)$ & $12,589(28)$ & $2,859(26)$ & $1,462(25)$ & $868(22)$ & 2,605 (18) \\
\hline
\end{tabular}

operatively (1-year levels: 17 vs. 12 Pain VAS, 70 vs. 79 EQ VAS, and 0.84 vs. 0.90 EQ-5D-SE). The adjusted estimated effect on 1 -year outcomes of having $\geq 4$ filled opioid prescriptions preoperatively, with opioid naive as reference, was 2.6 (CI 2.3-2.8) for Pain VAS, -3.9 (CI -4.1 to -3.7) for EQ VAS, and -0.02 (CI -0.02 to -0.02 ) for EQ-5D-SE (Table 6).

\section{Discussion}

Our main findings were in line with recent studies: preoperative opioid use may be a risk factor for worse outcomes in THR. A recent meta-analysis of 5 studies concluded a higher risk of revision for preoperative opioid users (OR 1.6, CI 1.2-1.7) (20). Another meta-analysis of PROMs after total joint replacement concluded that patients with preoperative opioid use experienced similar magnitude of improvement to patients with no filled opioid prescriptions but ended up at a worse level numerically on the scale (5). In addition to worse PROMs the preoperatively opioidtreated groups had higher rates of persistent opioid use in the postoperative period. In our study there was a markedly higher risk for revision and reoperation within 2 years with $\geq 4$ opioid prescriptions filled preoperatively. We could also identify a higher risk of adverse events within the first 90 days, although this effect was less pronounced. Regarding PROs, we also demonstrated that the group with $\geq 4$ opioid prescriptions ended up at a worse level 1 year postoperatively. This was especially evident regarding EQ VAS, where the estimated effect of $\geq 4$ opioid prescriptions preoperatively corresponded to a 3.9 points worse result. This equals $18 \%$ of the mean change for the whole population. Given the overall large improvements in pain and function following THR, single investigated patient factors or differences in treatment routines can be expected to be associated only with marginal effects on PROs. In the pursuit of finding modifiable factors that can lead to further improvement of THR outcomes, we believe the patient-reported results of this study are clinically important on a group level and may help guide patients before THR. A recent study of a population in the south of Sweden suggested that a large proportion of opioid prescriptions for OA patients is inappropriate according to current guidelines (21). There is a possibility that OA patients with inappropriate opioid use are put at higher risk of worse outcomes by receiving a treatment with little or no effect on their symptoms, while still being at risk of opioids' side effects and iatrogenic addiction. Misuse of prescribed opioids causing drug addiction has caused severe morbidity and suffering in North America. While this problem is currently not as great in Europe, this calls for care and vigilance in the use of opioids as prescription rates increase (22).

Our finding that women were treated with opioids to a higher degree than men among OA patients with a hip replace- 
Table 3. Analysis group 2: patient-reported outcomes. Patient characteristics at baseline grouped by number of filled opioid prescriptions among adult osteoarthritis patients with total hip replacement. Values are number of patients (\%)

\begin{tabular}{|c|c|c|c|c|c|c|}
\hline \multirow[b]{2}{*}{ Factor } & \multirow[b]{2}{*}{ Entire group } & \multicolumn{5}{|c|}{ Number of filled opioid prescriptions } \\
\hline & & 0 & 1 & 2 & 3 & $\geq 4$ \\
\hline Total observations & 64,015 & $36,641(57)$ & $8,595(13)$ & $4,657(7)$ & $3,046(5)$ & $11,076(17)$ \\
\hline \multicolumn{7}{|c|}{ 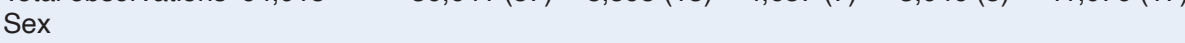 } \\
\hline Female & $36,240(57)$ & $19,320(53)$ & $5,180(60)$ & $2,840(61)$ & $1,873(61)$ & $7,027(63)$ \\
\hline Male & $27,775(43)$ & $17,321(47)$ & $3,415(40)$ & $1,817(39)$ & $1,173(39)$ & 4,049 (37) \\
\hline \multicolumn{7}{|l|}{ Age group } \\
\hline$\leq 39$ & $424(1)$ & $239(1)$ & $55(1)$ & $24(1)$ & $23(1)$ & $83(1)$ \\
\hline $40-44$ & $704(1)$ & $416(1)$ & $97(1)$ & $43(1)$ & $36(1)$ & $112(1)$ \\
\hline $45-49$ & $1,713(3)$ & 949 (3) & 240 (3) & $118(3)$ & 77 (3) & 329 (3) \\
\hline $50-54$ & $3,276(5)$ & $1,917(5)$ & $458(5)$ & $202(4)$ & $146(5)$ & $553(5)$ \\
\hline $55-59$ & $5,589(9)$ & $3,237(9)$ & 764 (9) & $390(8)$ & $262(9)$ & 936 (8) \\
\hline $60-64$ & $9,853(15)$ & $5,650(15)$ & $1,391(16)$ & $718(15)$ & $441(14)$ & $1,653(15)$ \\
\hline $65-69$ & $12,813(20)$ & $7,691(21)$ & $1,606(19)$ & 868 (19) & 568 (19) & $2,080(19)$ \\
\hline $70-74$ & $12,203(19)$ & 7,106 (19) & 1,658 (19) & $899(19)$ & $585(19)$ & $1,955(18)$ \\
\hline $75-79$ & $9,639(15)$ & $5,482(15)$ & $1,257(15)$ & $744(16)$ & $484(16)$ & $1,672(15)$ \\
\hline $80-84$ & $5,629(9)$ & $2,920(8)$ & $785(9)$ & $447(10)$ & $307(10)$ & $1,170(11)$ \\
\hline$\geq 85$ & $2,172(3)$ & $1,034(3)$ & $284(3)$ & $204(4)$ & $117(4)$ & $533(5)$ \\
\hline \multicolumn{7}{|l|}{ ASA score } \\
\hline 1 & $16,505(27)$ & $10,817(30)$ & $2,217(27)$ & $1,067(24)$ & $605(20)$ & $1,799(17)$ \\
\hline 2 & $36,933(59)$ & 20,828 (59) & $5,025(60)$ & $2,736(60)$ & $1,811(61)$ & $6,533(61)$ \\
\hline$\geq 3$ & $8,635(6)$ & $3,889(1)$ & $1,073(13)$ & 719 (16) & $550(19)$ & $2,404(22)$ \\
\hline \multicolumn{7}{|l|}{ BMI category } \\
\hline$\leq 24.9$ & $19,573(32)$ & $11,746(43)$ & $2,630(32)$ & $1,397(31)$ & $824(28)$ & $2,976(28)$ \\
\hline $25-29.9$ & $26,837(44)$ & $15,772(45)$ & $3,624(44)$ & $1,873(42)$ & $1,290(44)$ & $4,278(40)$ \\
\hline $30-34.9$ & 11,332 (19) & $5,984(17)$ & $1,487(18)$ & 904 (20) & $61621)$ & $2,341(22)$ \\
\hline$\geq 35$ & $3,389(6)$ & $1,473(4)$ & $454(6)$ & $292(7)$ & $194(7)$ & 976 (8) \\
\hline \multicolumn{7}{|l|}{ Surgical approach } \\
\hline Lateral & $30,178(48)$ & $17,476(48)$ & $4,109(48)$ & $2,185(47)$ & $1,380(46)$ & $5,028(46)$ \\
\hline Posterior & $33,317(52)$ & $18,844(52)$ & $4,413(52)$ & $2,441(53)$ & $1,651(54)$ & 5,968 (54) \\
\hline \multicolumn{7}{|l|}{ Fixation method } \\
\hline Cemented & $42,431(66)$ & $23,757(65)$ & $5,693(66)$ & $3,197(69)$ & $2,120(70)$ & $7,664(69)$ \\
\hline Uncemented & $11,682(18)$ & $7,144(19)$ & $1,533(18)$ & 754 (16) & 494 (16) & 1,757 (16) \\
\hline Hybrid & $1,346(2)$ & $758(2)$ & $165(2)$ & $94(2)$ & $63(2)$ & 266 (2) \\
\hline Reverse hybrid & 8,556 (13) & 4,982 (14) & $1,204(14)$ & 612 (13) & 369 (12) & 1,389 (13) \\
\hline \multicolumn{7}{|l|}{ Educational level } \\
\hline Low & $20,420(32)$ & $11,156(31)$ & 2,726 (32) & $1,556(34)$ & $1,079(36)$ & 3,903 (35) \\
\hline Middle & $26,483(42)$ & $14,992(41)$ & $3,553(42)$ & $1,952(42)$ & $1,279(42)$ & $4,707(43)$ \\
\hline High & $16,742(26)$ & $10,292(28)$ & $2,265(27)$ & 1,119 (24) & $665(22)$ & $2,401(22)$ \\
\hline \multicolumn{7}{|l|}{ Income level } \\
\hline Low & $15,058(24)$ & $7,782(21)$ & $1,956(23)$ & $1,151(25)$ & $821(27)$ & $3,348(30)$ \\
\hline Middle & $32,351(50)$ & $18,478(51)$ & $4,327(50)$ & $2,331(50)$ & $1,532(50)$ & $5,683(51)$ \\
\hline High & 16,565 (26) & $10,351(28)$ & $2,309(27)$ & $1,172(25)$ & 692 (23) & 2,041 (19) \\
\hline
\end{tabular}

groups. A recent New Zealand study of TJR patients reported increased risk of postoperative chronic opioid use in patients with socioeconomic deprivation and low income, as well as indicating higher education as a protective factor against continued opioid use (25). This further supports socioeconomics as an important factor to consider, both when prescribing analgesics and when risk stratifying patients eligible for THR.

\section{Limitations and strengths}

A limitation of our study may be that dose and time intervals of opioid prescriptions have not been specified. In the dataset we used, more detailed information was not available and requesting it would have required a completely new database to be formed from the quality registers and health data registers. We still considered our available level of detail on prescriptions worth exploring, even though further studies should probably include more detailed data on prescribed amounts of opioids. We defined $\geq 4$ filled prescriptions as a likely level where long-term or repeated opioid use has occurred, and this group accounted for $18 \%$ of our study population. With greatly varying prescribing patterns between countries, we did not have a direct comparison for this number. A recent study from Denmark classified 24\%

ment corresponds to the general prescribing of opioids in Sweden, with a surplus of women in the general population filling at least 1 opioid prescription in any given year (23). A recent national observational study from the Unites States also showed that women received more opioid prescriptions than men but found no statistically significant difference between the sexes in opioid prescription, when adjusting for socioeconomics and comorbid conditions. The authors hypothesized that women's higher opioid utilization could be due to worse socioeconomic profile, comorbid conditions, and a general propensity to be subjected to more healthcare (24). The notion of socioeconomic profile affecting opioid prescribing could hold true for our population of THR patients as well, with higher prevalence of having $\geq 4$ opioid prescriptions filled preoperatively in the low education level and low-income level of patients going through hip or knee replacement as opioid users in the month preceding surgery (26). This could be a relevant comparison, with Denmark having a similar or slightly lower number of opioid-treated per 1,000 inhabitants compared with Sweden (27). Hence, our measure of opioid use may be a robust and easily available surrogate for the purpose of investigating the influence of opioid treatment on outcomes in THR. However, we cannot draw any detailed conclusions about dose-response relationships as information on the actual amount of consumed drugs is lacking. Nevertheless, across the exposure categories of our crude measure of number of filled prescriptions, there was a suggested "dose-response" trend for most outcomes. We did not have information on underlying diagnosis for the individual prescriptions and therefore cannot tell if the opioid treatment was the result of 
Table 4. Revision, reoperation, and adverse events among adult osteoarthritis patients with total hip replacement. Adjusted odds ratios (OR) weighted with inverse probability weights and adjusted for age, sex, BMI (continuous), ASA score, Elixhauser comorbidity, income level, educational level, fixation method, and surgical approach

\begin{tabular}{|c|c|c|c|}
\hline $\begin{array}{l}\text { Number of } \\
\text { prescriptions } \\
\text { of any opioid }\end{array}$ & $\mathrm{n}(\%)$ & OR (95\% Cl) & $\begin{array}{c}\text { Adjusted } \\
\text { OR }(95 \% \mathrm{Cl})\end{array}$ \\
\hline \multicolumn{4}{|c|}{ Revision within 2 years, $n=1,024$ (1.3) } \\
\hline 0 & $482(1.1)$ & Ref. & Ref. \\
\hline 1 & 139 (1.3) & $1.2(1.0-1.5)$ & $1.2(1.1-1.3)$ \\
\hline 2 & $82(1.4)$ & $1.3(1.0-1.7)$ & $1.3(1.1-1.4)$ \\
\hline 3 & $60(1.5)$ & $1.5(1.1-1.9)$ & $1.3(1.2-1.4)$ \\
\hline$\geq 4$ & $261(1.8)$ & $1.7(1.4-2.0)$ & $1.4(1.3-1.6)$ \\
\hline \multicolumn{4}{|c|}{ Any reoperation within 2 years, $n=1,491(1.9)$} \\
\hline 0 & $702(1.6)$ & Ref. & Ref. \\
\hline 1 & $193(1.8)$ & $1.2(1.0-1.4)$ & $1.1(1.0-1.2)$ \\
\hline 2 & $125(2.1)$ & $1.4(1.1-1.7)$ & $1.3(1.2-1.4)$ \\
\hline 3 & $98(2.5)$ & $1.6(1.3-2.0)$ & $1.5(1.4-1.6)$ \\
\hline$\geq 4$ & $373(2.5)$ & $1.7(1.5-1.9)$ & $1.4(1.3-1.5)$ \\
\hline \multicolumn{4}{|c|}{ Adverse event within 90 days, $n=5,821$ (7.2) } \\
\hline 0 & $2,899(6.4)$ & Ref. & Ref. \\
\hline 1 & $777(7.2)$ & $1.1(1.0-1.2)$ & $1.1(1.1-1.1)$ \\
\hline 2 & $423(7.2)$ & $1.1(1.0-1.3)$ & $1.0(1.0-1.1)$ \\
\hline 3 & 339 (8.7) & $1.4(1.2-1.6)$ & $1.2(1.2-1.3)$ \\
\hline$\geq 4$ & $1,383(9.4)$ & $1.5(1.4-1.6)$ & $1.2(1.2-1.3)$ \\
\hline
\end{tabular}

OA or other diagnosis. There is also a risk of misclassification due to routine preoperative prescription of opioid analgesics intended for postoperative use at some arthroplasty centers. However, in a sensitivity analysis there was no clear spike in opioid prescriptions in the month prior to surgery, indicating that this possible routine is not influential on our results. Another limitation is the fact that the study design is based on already collected data and limited to available variables in the registers used, making it possible that other unknown factors could influence the results. We had a reasonably broad range of potential confounding variables available, and in order to use this information effectively to reduce potential bias, we performed a propensity score weighting. Even though several variables used to calculate the propensity score were collected after the studied opioid treatments occurred (i.e., at the surgery date), we expect them to be fairly consistent over the study period and thus may be used as a reasonable proxy for pre-treatment values. The major strengths of this study were the large number of included cases and high coverage of the PROM program, with all Swedish units performing THRs reporting and with a response rate of over $80 \%$ and $70 \%$ of patients completing both preoperative and 1-year postoperative questionnaires. The SHAR covers almost $98 \%$ of THRs performed due to OA in the years studied, making it a highly valid representation of the Swedish THR population (12).

\section{Possible mechanisms}

It is unclear how opioid use could be associated with worse outcomes after THR, but some possible mechanisms can be suggested. The side effects of opioids on cognitive and motor skills combined with emesis, constipation, and confusion could cause prolonged postoperative stay and delayed mobilization $(28,29)$. The negative effect of opioids on the immune system may increase the susceptibility to postoperative infections; the preoperative use of opioid medication has been suggested to be an independent risk factor for periprosthetic joint infection (30). Another possible mechanism for negative effects of opioids on outcome after joint replacement is opioid-induced hypersensibility $(\mathrm{OIH})$. This is defined as a state of sensitization to nociceptive stimuli paradoxically caused by exposure to opioid analgesics. OIH can cause both exacerbation of the pain meant to be treated in the first place and more intense pain in completely different parts of the body caused by old or new painful stimuli, such as surgery. Another troubling dimension of OIH is that the pain does not respond favorably to increased opioid doses but can conversely be aggravated by increased doses, accompanied by other adverse effects of higher opioid doses (31). In the setting of THR this could seriously impair early mobilization because of difficulties in treating severe pain pharmacologically. Further, patients with OIH are more likely to need higher doses of fast-acting opioids postoperatively, with increased risk of intestinal obstruction/bowel dysfunction, urinary retention, nausea/vomiting, cognitive impairment, and motor impairment (32). It is also possible that opioid treatment is associated with other unknown factors that may be important for the outcome of THR. For example, it has been proposed that individuals with a tendency to catastrophic thinking and a lack of coping mechanisms are more likely to be treated with opioids (33). 
Table 6. Effect estimates of preoperative opioid treatment on PROM among adult osteoarthritis patients with total hip replacement

\begin{tabular}{|c|c|c|c|c|c|}
\hline \multirow{2}{*}{$\begin{array}{l}\text { 1-year patient-reported } \\
\text { outcome measure }\end{array}$} & \multicolumn{5}{|c|}{ No. of filled opioid prescriptions } \\
\hline & 0 & 1 & 2 & 3 & $\geq 4$ \\
\hline \multicolumn{6}{|c|}{ Estimated effect, unadjusted $(95 \% \mathrm{Cl})$} \\
\hline Pain VAS & Ref. & $2.2(1.8$ to 2.6$)$ & $3.2(2.7$ to 3.7$)$ & 3.6 (3.0 to 4.3 ) & 5.5 (5.1 to 5.9$)$ \\
\hline EQ VAS & Ref. & $-2.9(-2.4$ to -3.3$)$ & $-4.4(-3.8$ to -5.0$)$ & $-5.5(-4.8$ to -6.2$)$ & $-9.6(-9.2$ to -10.1$)$ \\
\hline EQ-5D SE & Ref. & $-0.02(-0.02$ to -0.02$)$ & $-0.03(-0.03$ to -0.03$)$ & $-0.03(-0.03$ to -0.03$)$ & $-0.06(-0.06$ to -0.06$)$ \\
\hline \multicolumn{6}{|c|}{ Estimated effect, adjusted $(95 \% \mathrm{Cl})^{\text {a }}$} \\
\hline Pain VAS & Ref. & $1.2(1.0$ to 1.4$)$ & $1.6(1.4$ to 1.9$)$ & $1.6(1.4$ to 1.8$)$ & 2.6 (2.4 to 2.8$)$ \\
\hline EQ VAS & Ref. & $-1.3(-1.1$ to -1.5$)$ & $-1.6(-1.4$ to -1.9$)$ & $-2.3(-2.0$ to -2.5$)$ & $-3.9(-3.7$ to -4.1$)$ \\
\hline EQ-5D SE & Ref. & $-0.01(-0.00$ to -0.01$)$ & $-0.01(-0.01$ to -0.01$)$ & $-0.01(-0.01$ to -0.01$)$ & $-0.02(-0.02$ to -0.02$)$ \\
\hline
\end{tabular}

a Adjusted values weighted with inverse probability weights and adjusted in respective regression analysis for preoperative pain VAS, EQ VAS, and EQ-5D SE, respectively, in addition to age, sex, ASA score, Charnley category, income level, educational level, and incidence of antidepressant medication, or medication indicating chronic pain, 1 year preoperatively.

For abbreviations, see Table 5.

\section{Conclusions}

Use of opioids in the preoperative period may be an individual risk factor, or a marker of other underlying risk factors, for complications and worse outcomes after THR. Assessment of opioid use should be included in the risk stratification of the individual patient eligible for THR, just as smoking, obesity, or other comorbidities are. With recent guidelines and reviews indicating a very limited effect of opioids on pain and function in chronic pain, treatment of pain with opioids should be avoided in OA patients.

JS, OR, and EB conceived and planned the study. EB, FN, OR, and JS planned and performed the statistical analyses. All authors discussed the results and contributed to writing the manuscript, which was drafted by JS.

Acta thanks Maaike Gademan and Brian Hallstrom for help with peer review of this study.

1. Rajamäki T J, Puolakka P A, Hietaharju A, Moilanen T, Jämsen E. Use of prescription analgesic drugs before and after hip or knee replacement in patients with osteoarthritis. BMC Musculoskelet Disord 2019; 20: 427. doi.org/10.1186/s12891-019-2809-4

2. Bannuru R R, Osani M C, Vaysbrot E E, Arden N K, Bennell K, Bierma-Zeinstra S M A, et al. OARSI guidelines for the non-surgical management of knee, hip, and polyarticular osteoarthritis. Osteoarthritis Cartilage 2019; 27: 1578-89. doi.org/10.1016/j.joca.2019.06.011

3. Thorlund J B, Turkiewicz A, Prieto-Alhambra D, Englund M. Opioid use in knee or hip osteoarthritis: a region-wide population-based cohort study. Osteoarthritis Cartilage 2019; 27: 871-7. doi.org/10.1016/j. joca.2019.01.005

4. Cozowicz C, Olson A, Poeran J, Mörwald E E, Zubizarreta N, Girardi F P, et al. Opioid prescription levels and postoperative outcomes in orthopedic surgery. PAIN 2017; 158: 2422-30. doi.org/10.1097/j. pain. 0000000000001047

5. Goplen C M, Verbeek W, Kang S H, Jones C A, Voaklander D C, Churchill T A, et al. Preoperative opioid use is associated with worse patient outcomes after total joint arthroplasty: a systematic review and meta-analysis. BMC Musculoskelet Disord 2019; 20: 234. 10.1186/ s12891-019-2619-8
6. Shadbolt C, Schilling C, Inacio M C, Abbott J H, Pryymachenko Y, Wilson R, et al. Opioid use and total joint replacement. Curr Rheumatol Rep 2020; 22: 58. doi.org/10.1007/s11926-020-00929-0

7. Ludvigsson J F, Otterblad-Olausson P, Pettersson B U, Ekbom A. The Swedish personal identity number: possibilities and pitfalls in healthcare and medical research. Eur J Epidemiol 2009; 24: 659-67. doi. org/10.1007/s10654-009-9350-y

8. Ludvigsson J F, Andersson E, Ekbom A, Feychting M, Kim J L, Reuterwall C, et al. External review and validation of the Swedish national inpatient register. BMC Public Health 2011; 11: 450. doi. org/10.1186/1471-2458-11-450

9. Elixhauser A, Steiner C, Harris D R, Coffey R M. Comorbidity measures for use with administrative data. Med Care 1998; 36: 8-27. doi. org/10.1097/00005650-199801000-00004

10. Bülow E. coder: An R package for code-based item classification and categorization. J Open Source Software 2020; 5(56). https://doi. org/10.21105/joss.02916

11. Ludvigsson J F, Svedberg P, Olén O, Bruze G, Neovius M. The longitudinal integrated database for health insurance and labour market studies (LISA) and its use in medical research. Eur J Epidemiol 2019; 34: 42337. doi.org/10.1007/s10654-019-00511-8

12. Kärrholm J, Rogmark C, Nauclér E, Nåtman J, Vinblad J, Mohaddes M, et al. Swedish Hip Arthroplasty Register: Annual report 2019; 2020.

13. Burström K, Sun S, Gerdtham U-G, Henriksson M, Johannesson M, Levin L-Å, et al. Swedish experience-based value sets for EQ-5D health states. Qual Life Res 2014; 23: 431-42. doi.org/10.1007/s11136013-0496-4

14. Newgard C D, Hedges J R, Arthur M, Mullins R J. Advanced statistics: the propensity score - a method for estimating treatment effect in observational research. Acad Emerg Med 2004; 11: 953-61. doi.org/10.1197/j. aem.2004.02.530

15. Goltz D E, Ryan S P, Howell C B, Attarian D, Bolognesi M P, Seyler T M. A weighted index of Elixhauser comorbidities for predicting 90-day readmission after total joint arthroplasty. J Arthroplasty 2019; 34: 85764. doi.org/10.1016/j.arth.2019.01.044

16. Ferguson R J, Silman A J, Combescure C, Bulow E, Odin D, Hannouche $\mathrm{D}$, et al. ASA class is associated with early revision and reoperation after total hip arthroplasty: an analysis of the Geneva and Swedish Hip Arthroplasty Registries. Acta Orthop 2019; 90: 324-30. doi.org/10.1 080/17453674.2019.1605785

17. Weiss R J, Kärrholm J, Rolfson O, Hailer N P. Increased early mortality and morbidity after total hip arthroplasty in patients with socioeconomic disadvantage: a report from the Swedish Hip Arthroplasty Register. Acta Orthop 2019; 90: 264-9. doi.org/10.1080/1453674.2019.1598710 
18. Rolfson O, Donahue G S, Hallsten M, Garellick G, Kärrholm J, Nemes S. Patient-reported outcomes in cemented and uncemented total hip replacements. Hip Int 2016; 26: 451-7. doi.org/10.5301/hipint.5000371

19. Greene M E, Rolfson O, Gordon M, Annerbrink K, Malchau H, Garellick G. Is the use of antidepressants associated with patientreported outcomes following total hip replacement surgery? Acta Orthop 2016; 87: 444-51. doi.org/10.1080/17453674.2016.1216181

20. Chen L, Wang Q, Li D, Chen C, Li Q, Kang P. Meta-analysis of retrospective studies suggests that the pre-operative opioid use is associated with an increased risk of adverse outcomes in total hip and or knee arthroplasty. Int Orthop 2021. doi.org/10.1007/s00264-021-04968-9. doi. org/10.1007/s00264-021-04968-9

21. Thorlund J B, Turkiewicz A, Prieto-Alhambra D, Englund M. Inappropriate opioid dispensing in patients with knee and hip osteoarthritis: a population-based cohort study. Osteoarthritis Cartilage 2020; 28: 146-53. doi.org/10.1016/j.joca.2019.10.004

22. van Amsterdam J, van den Brink W. The misuse of prescription opioids: a threat for Europe? Curr Drug Abuse Rev 2015; 8: 3-14. http:// dx.doi.org/10.2174/187447370801150611184218

23. Läkemedelsverket. Förskrivning av opioider i Sverige: Läkemedel, doser och diagnoser. Rapport från Läkemedelsverket; 2020. Dnr: 4.3.12018-102265.

24. Agnoli A, Jerant A, Franks P. Prescription opioids and patient sex: a national cross-sectional study. J Womens Health (Larchmt) 2021; 30: 29-35. doi.org/10.1089/jwh.2019.8234

25. Pryymachenko Y, Wilson R A, Abbott J H, Dowsey M M, Choong P F M. Risk factors for chronic opioid use following hip and knee arthroplasty: evidence from New Zealand population data. J Arthroplasty 2020; 35: 3099-107.e3014. doi.org/10.1016/j.arth.2020.06.040
26. Jørgensen C C, Petersen M, Kehlet H, Aasvang E K. Analgesic consumption trajectories in 8975 patients 1 year after fast-track total hip or knee arthroplasty. Eur J Pain 2018; doi.org/10.1002/ejp.1232. doi. org/10.1002/ejp.1232

27. Jarlbaek L. Opioid prescribing habits differ between Denmark, Sweden and Norway - and they change over time. Scand J Pain 2019; 19: 491-9. doi.org/10.1515/sjpain-2018-0342

28. Petre B M, Roxbury C R, McCallum J R, Defontes K W, Belkoff S M, Mears S C. Pain reporting, opiate dosing, and the adverse effects of opiates after hip or knee replacement in patients 60 years old or older. Geriatr Orthop Surg Rehabil 2012; 3: 3-7. doi.org/10.1177/2151458511432758

29. Sjøgren P, Thomsen A B, Olsen A K. Impaired neuropsychological performance in chronic nonmalignant pain patients receiving long-term oral opioid therapy. J Pain Symptom Manage 2000; 19: 100-8. doi. org/10.1016/s0885-3924(99)00143-8

30. Bell K L B A, Shohat N M D, Goswami K M D, Tan T L M D, Kalbian I B A, Parvizi J M D F. Preoperative opioids increase the risk of periprosthetic joint infection after total joint arthroplasty. J Arthroplasty 2018; 33: 3246-51.e3241. http://dx.doi.org/10.1016/j.arth.2018.05.027

31. Lee M, Silverman S M, Hansen H, Patel V B, Manchikanti L. A comprehensive review of opioid-induced hyperalgesia. Pain Physician 2011; 14: $145-61$.

32. Hina N, Fletcher D, Poindessous-Jazat F, Martinez V. Hyperalgesia induced by low-dose opioid treatment before orthopaedic surgery: an observational case-control study. Eur J Anaesthesiol (EJA) 2015; 32: 255-61. doi.org/10.1097/eja.0000000000000197

33. Sturgeon J A, Zautra A J. Resilience: a new paradigm for adaptation to chronic pain. Curr Pain Headache Rep 2010; 14: 105-12. doi. org/10.1007/s11916-010-0095-9 